\title{
The Physiological Standardization of Drugs
}

How the Strength of Drugs is Determined and the Proper Dose Found

By Lewis Davis, M. S.

IT is a curious fact that, to the average person, all a test frog weighing one million times that amount (20 medicinal agents are shrouded in a veil of mystery. Take the term "drug," for example, and it unconsciously brings to the lay mind the childhood conception of the simmering pot in the deep wood filled with strange roots and herbs, and attended by the witch with her peaked hat and crooked staff. Only to such hardy adventurers as the physician and the pharmacist has been left the task of penetrating this maze. The ordinary mortal is content to escape by heroically taking doses of bitter-tasting "medicines" in his illness, or stoically submitting to hypodermic injections, regardless of the origin or value of the material employed.

Fortunately, science does not share in this attitude. Careful investigation has not only succeeded in establishing the composition and therapeutic action of many drugs, but has set up certain standards of activity to which they must conform. The modern physician no longer prescribes drugs, trusting to good fortune that
they contain a requisite amount of active principle. These have all been assayed in the laboratory by their actions on test animals with a degree of delicacy far surpassing the most refined chemical or physical means. When it is considered that 0.00002 gramme of the active principle of Strophanthus seeds will cause the death of grammes), the extreme sensitiveness required of a suitable method of standardization is readily apparent. In fact, of such extreme delicacy, but are more usually of no value.

It might be well to ask, what are these substancesdrugs-which are so highly potent in their effects? A popular definition of a "drug" might be given as any substance-animal, vegetable, or mineral-used in the preparation of medicine, or in the cure of disease. This would include chemicals, the alkaloids, vaccines, serums, certain gland products, and finally, those plant substance of undetermined chemical nature which are capable, upon introduction into the body, of producing certain distinet efects. Properly speaking it is these last two vativesuld be designated as "drugs."

While the plant derivatives, particularly the heart tonics, have been known to the scientific world for decades, our knowledge of the gland products and their actions has all come to us within the past fifteen years Two of these, in particular, command our attentionproducts from the suprarenal gland and those from the pituitary gland.
Surmounting each kidney usually, or lying very close to it, we find the right and left adrenal glands, the latter being usually the larger. They differ in shape from eac other, and the right capsule having a flattened triangular form, the left a crescent shape. There are also variations from these forms. In the human they weigh about four rrammes and are nearly as large at birth as in ar irth as in adult life. In color they are dark red, or sometimes yellowish red.
One or both capsules may be absent, although this is One or both caps
rarely the case.

The glands are smaller in advanced age, and it is said
The the case. that negroes have larger ones than Caucasians, which fact no doubt bears some relation to the pigmentation of the skin.

When sectioned, they are seen to consist principally of two parts, an outer firm portion (cortical), and an parts are usually quite definitely marked, but it is difficult to separate them perfectly since they are more or less closely "dove-tailed" into each other.

Removal of the suprarenal bodies in animals is speedily followed by symptoms of extreme muscular prostration and within a few days by death. For years a puzzle to the physiologist and physician, it came as a surprising discovery that some of the constituents of the suprarenal

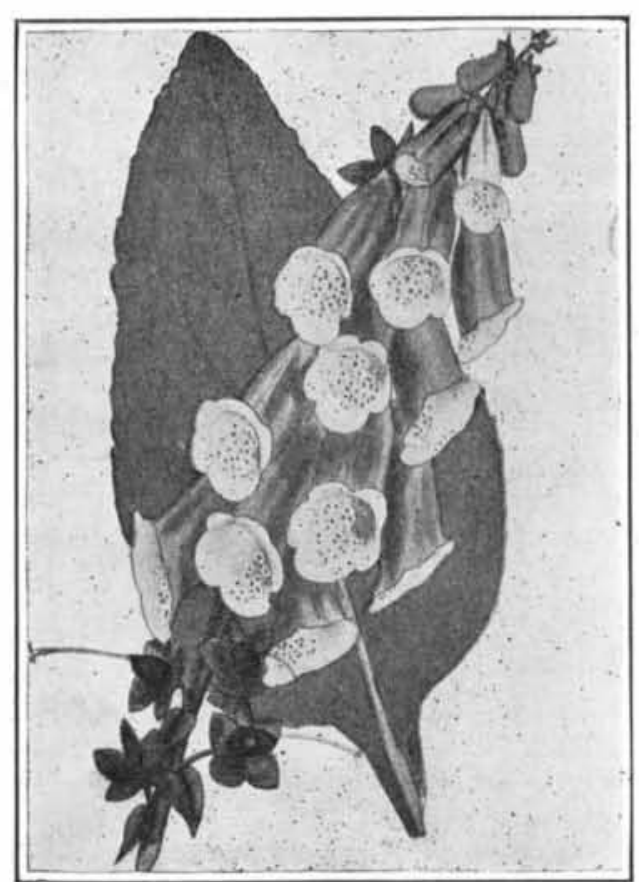

Fig. 1.-Foxglove

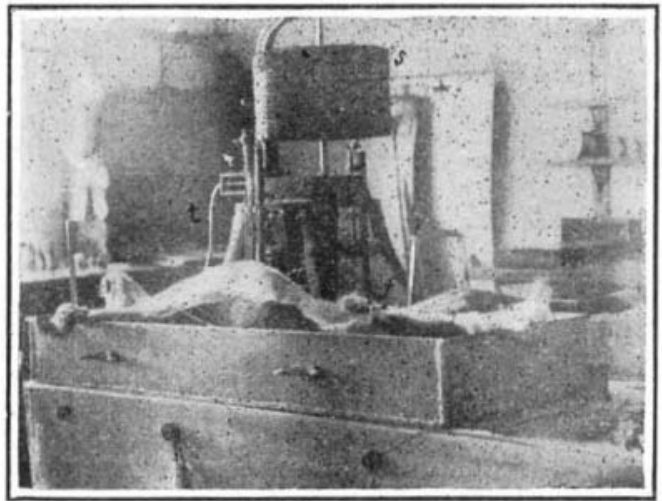

Fig. 5.-Dog connected with kymograph as used in assaying adrenalin and pituitary extracts.

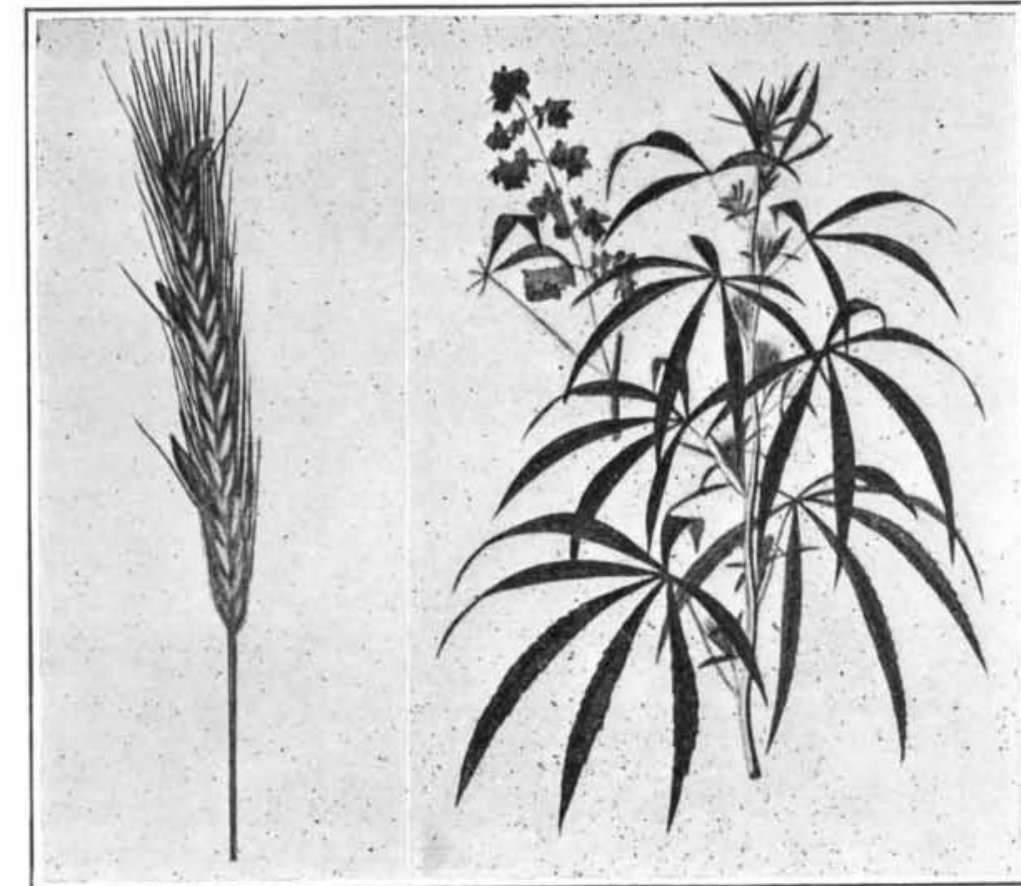

Fig. 2.-Ergot in rye.

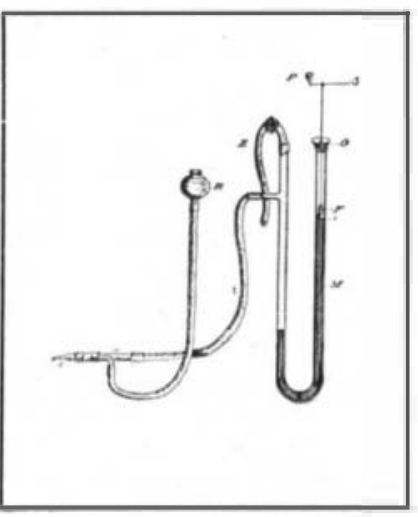

Fig. 5a.-Recording mer. cury manometer and canule.

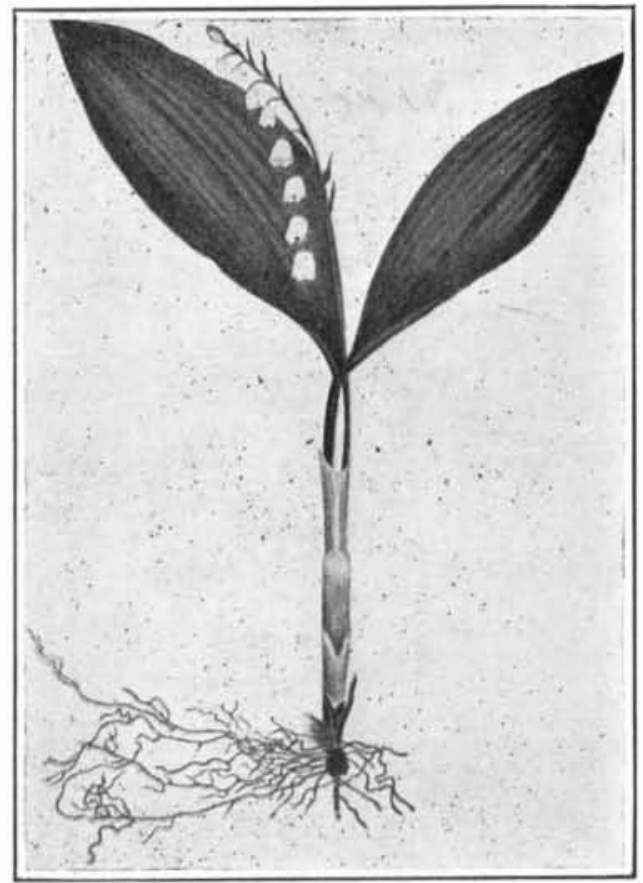

Fig. 3.-Lily-of -the-valley

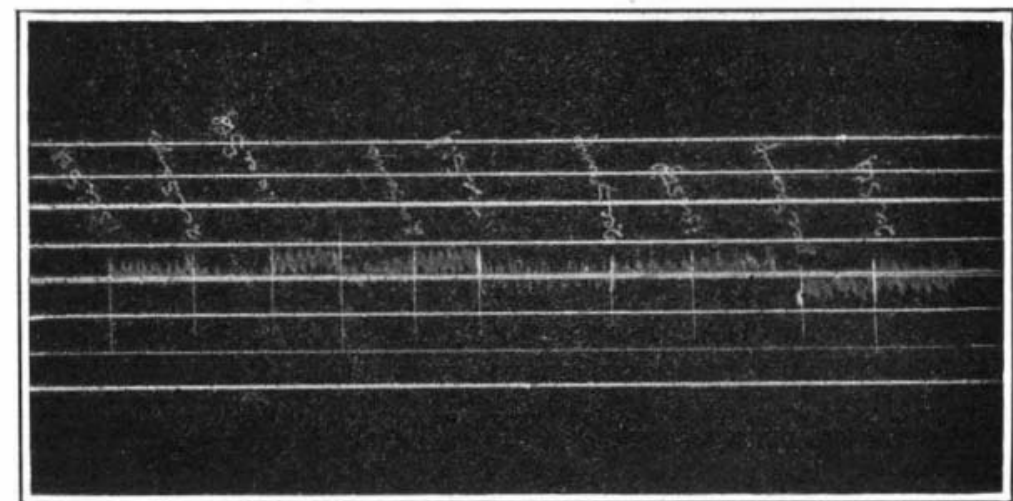

Fig. 8. - Tracing made by the kymograph, showing rise and fall of blood pressure, due to adrenalin chloride solution.

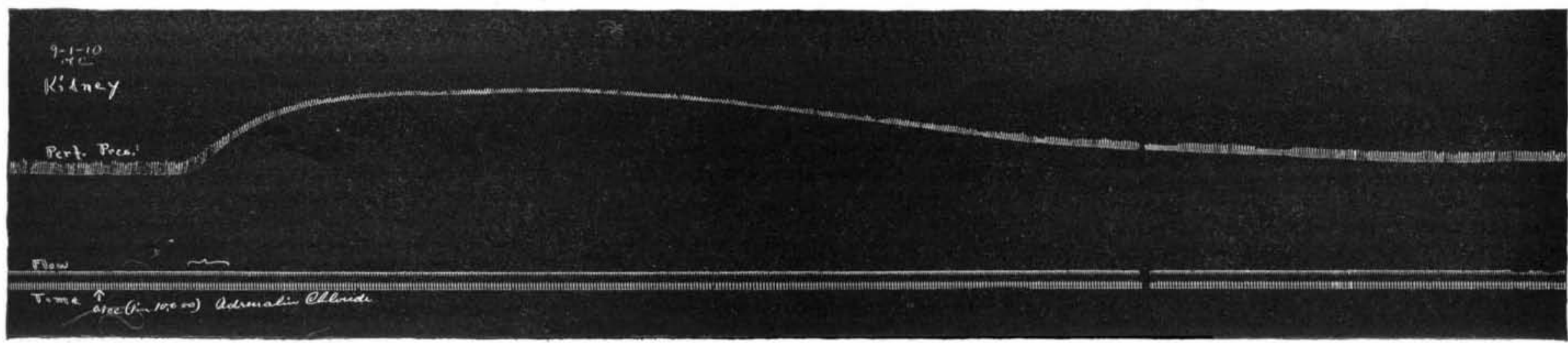

Fig. 10. - Tracings obtained with kymographion of Fig. 2 in testing unknown samples. 


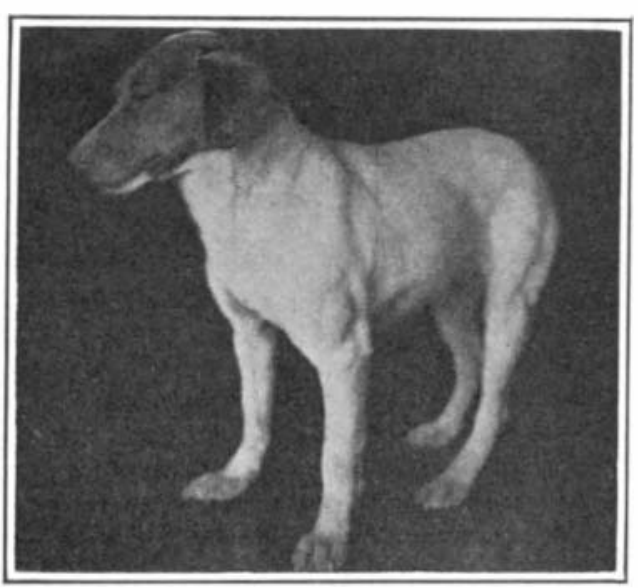

Fig. 4.-Dog showing characteristic intoxication of cannabis.

gland had a remarkable influence upon blood pressure Few discoveries in medicine have been of greater import ance than the announcement in 1897, that the suprarenal gland contained a certain substance which had the wonderful power of lessening, or even preventing, hemorrhag by virtue of its local or constricting action on the bloo vessels. In other words, here is an agent at the command of the physician which diminishes the flow of blood by simply, so to speak, "stopping it up." As would be expected, extracts and various products of this gland soon pected, extracts and various products of this gland soo came into use for producing bloodless operations in ey of hemorrhage, and for increasing the blood pressure. Careful scientific investigation has resulted not only in the preparation of the active constituent of the gland in a chemically pure form, but more recently a synthetic product has come upon the market, identical in all its remarkable properties with the natural product obtained from beef.

The natural material, known under various names, as "adrenalin." "suprarenin," etc., is, when pure, prac tically white in color, and composed of microscopic crystals of various forms. These chemically pure crystals (of "adrenalin") serve as a standard for the assay of products of the suprarenal glands, and the strength of any product is stated in terms of the standard-as, for example, "Solution of Adrenalin" 1-10,000, etc.

Although very recently a chemical method has been proposed for the assay of adrenalin and similar products, the physiological method is used almost entirely. Various animals may be used for this purpose, but the best results animals may be used for this purpose,
are obtained with medium-sized dogs.

The physiological determination of the strength of products from the suprarenal and of the pituitary gland - these latter substances will be discussed later-is al most identical. The method depends upon the fact that when properly diluted doses of these drugs are injected directly into the circulatory system of test animals $(\operatorname{dog} s)$ there is produced a definite, characteristic rise in the blood pressure of the animal which soon comes back to blood pressure of the animal which soon comes back to
normal. The test animal is first anæsthetized completely, normal. The test animal is first anæsthetized completely,
after which the hair is clipped from the throat and the tissues severed. A glass "cannula," as it is called, or connecting tube of suitable size is now tied in the common carotid artery, which, in turn, is connected with a tube filled with sodium carbonate (soda ash) or other solution, to prevent the blood from clotting. The latter tube is attached to a manometer or "U" tube containing mercury. A schematic representation of a recording mercury cury. A schematic representation of a recording mercury
manometer and its connection with the artery is shown in Fig. 5a. C represents the "cannula" which is inserted in the artery previously clamped. On releasing the clamp, the pressure of the blood is communicated through the liquid in $t$ to the column of mercury in the manometer $M$, until the weight of the column which is forced up, shown by the difference in level between 1 and 2 counterbalances the pressure of the blood. The limb of the U tube in which the mercury rises carries a float $(f)$ of aluminium, hard rubber, or some other substance lighter than the mercury. This float, in turn, carries a stiff wire which plays through an opening in the cap $(G)$ of the tube. The free end of this wire bears a needle $(P)$ or more usually a bent metal arm, which marks a tracing on a revolving sheet $S$. The latter may be plain, white, or sensitized paper, but is more often smoked paper, and the record scratched out by the metal pen.

Such a complete recording apparatus, known as the "kymographion." and its connections to the test dog are shown in Fig. 5, as used in a large commercial laboratory for the routine standardization of products from the suprarenal and pituitary glands. The method, in brief, is as follows:

A definite quantity of the standard solution is injectfid into the femoral vein (Fig. 5), and with the kymographion in motion, the maximum rise in blood pressure is communicated through the tube $t$ and recorded on the revolving sheet $(s)$. The pressure is now allowed to return to normal, after which, varying amounts of the solution are

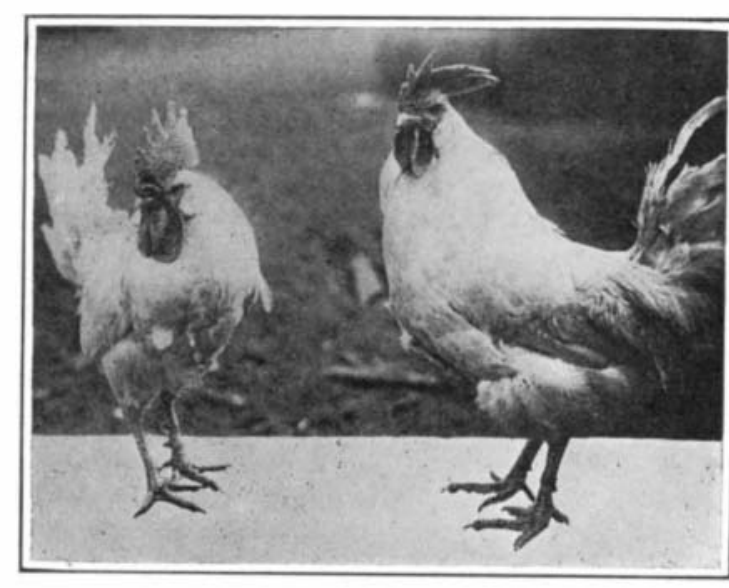

Fig. 6.-Roosters used in testing strength of ergot.

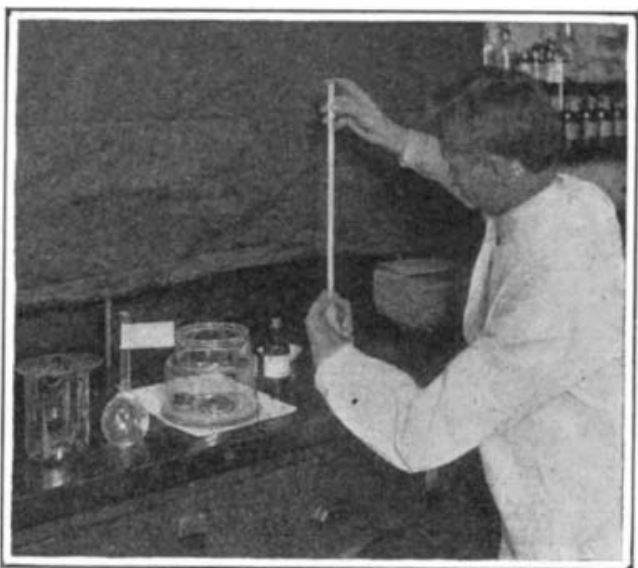

Fig. 7.-Testing the strengths of the heart tonics by their actions on frogs. similarly injected until the resultant increase of pressure as recorded by the needle is equal to that produced by the known amount of the standard. For example, if it takes three cubic centimeters of the unknown to produce the same rise in blood pressure as that caused by on cubic centimeter of the standard, then the sample is said to be "331/3 per cent of standard," etc. A tracing of this sort is shown in Fig. 10, while Fig. 8 shows the sudden rise and slow return of pressure due to an injection of adrenalin chloride ( 1 in 100,000 parts).

A s already mentioned, the usual method of standardizing extracts of the pituitary gland is identical with that described for adrenalin. The pituitary body or "hypophysis cereberi" as it is scientifically termed, is even a greater mystery than the suprarenal glands.

Hidden away in the very middle of the skull in a little bony cup (sella turcica), better protected than any othe oland in the body, and very inaccessible, is the little body known to anatomists as the pituitary body, believed for

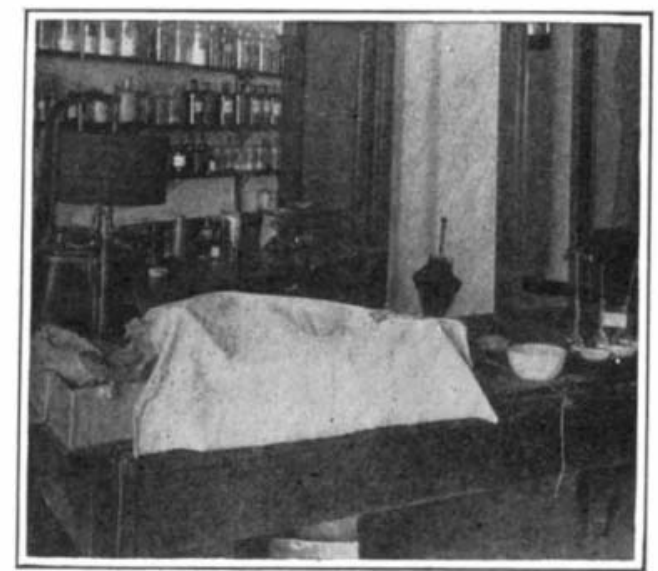

Fig. 9.-View of laboratory fitted for drug standardization.

a long time, until 1886, to be void of all physiological value, in fact, a sort of useless appendix of the brain Some of the older writers ascribed to it the function of secreting mucus, hence the term "pituitary body"; others ascribe to it manifold functions. In the human and in sheep it weighs about 0.5 grammes, in eattle 2.5 to 3 grammes. In shape it is oval, and when fresh it has a pinkish color.

If we were to section the pituitary gland, we would see plainly two definitely marked parts, but histologists tell us that it is composed of three parts-anterior, posterior and intermediate. It is usually described as consisting of two parts-the larger or "anterior lobe," and the much smaller "posterior lobe." The "posterior lobe" arises as an outgrowth from the floor of the brain and it is this portion which contains physiologically active substances of considerable therapeutic importance. The active principle, known as "pituitrin," "hypophysin," etc., also has the power of raising the blood pressure similar to that of adrenalin, but to a more marked degree. In addition, it has the remarkable property of causing contractions of the uterus, and within the last few years has come to be of great assistance during child-birth, in cases of uterine insufficiency. The drug has also the power of stimulating the secretion of urine and especially of milk.

Disturbance of the pituitary due to disease or otherwise may bring about the conditions known as "acromegaly" and "gigantism." In the former case there is an enlargement of the bones of the face and extremities, while in the latter there is an overgrowth in the whole skeleton. Both in pituitrin, but particularly so in adrenalin, there is a more or less clearly defined active principle upon which the physiologic assay can be based. There are, however, two very important drugs which have no such constituent that can be utilized to base a method upon. These are ergot and cannabis.

Under certain conditions rye may become affected with a black smut or fungus growth which is known as the "ergot" of rye (Fig. 2). Almost all of the ergot of com- merce comes from Russia and Siberia. Aside from its use to stimulate the heart and to increase the blood pressure, the alcoholic (fluid) extract of the druy is employed to assist contractions of the uterus in child-birth in the same way as pituitrin.

Considerable research on the subject has failed to bring about any agreement as to the active principle or principles of the drug. Blood pressure experiments gave too variable results to be relied upon, while its contraction of the muscle of the uterus also proved unreliable. Not baffled by this, the pharmacologist has cast about and found the desired subject in our shrill announcer of the dawn - the rooster. The physiological testing of ergot depends upon a characteristic blackening or blanching which it produces in the comb and wattles of selected cocks, when the drug or its preparations are internally administered in suitably sized doses.

The test fowls are normal, thoroughbred, white Leghorn cocks, twelve to eighteen months old, which have been allowed to fast for twenty-four hours. The animals should not be used for a longer period than six months, and not oftener than once a month. 'The standard used is a fluid extract of the drug, made up according to the "U. S. Pharmacopoia," and which by repeated tests has been shown to possess the average activity of, at least, ten samples of first class, commercial ergot. The alcohol in both the standard and the unknownsamples is removed by evaporation, and both converted into as nearly the same condition as possible. The alcohol-free samples were formerly introduced into the crops of several of the test fowls through a soft rubber tube in doses of 15 cubic centimeters per kilo of body weight. Now, however, it is more usual to inject a smaller dose directly into the breast muscle. By varying the size of dose and repeated trials, the amount of the unknown is determined which will produce the same degree of blackening in the parts as the test dose of the standard. (See Fig. 6.)

Very rarely is intoxication induced for such a worthy purpose as in the standardization of cannabis. "Cannabis Sativa," or, as it is better known, "Indian Hemp," is extensively employed in the treatment of insomnia, neuralgia, headache and hysteria. The dried, flowering tops of the plant are used, and careful investigation has shown the domestic variety of the drug, "American Hemp," to be fully as efficient as the imported product from India.

The active constituents of Cannabis are even less understood than in the case of ergot, consequently chemical standardization is out of the question. Here again, a physiological method is made use of: with healthy, medium-sized, short-haired dogs, young, but full grown, as the test animals. The standard used is a U. S. Pharmacopoia extract of cannabis having the average activity of not less than ten samples of first-class, commereial drugs, and the standard test dose is 0.010 gramme per kilo body weight.

In testing, at least two dogs, susceptible to the drug, are used, one of which receives the standard, and the other the unknown sample. These are administered internally in a small capsule by drawing the dog's tongue forward between the teeth with the left hand, and placing the capsule on the back part of the tongue with the right hand. On quickly releasing the tongue, the capsule is swallowed with ease. To aid in absorbing the drug, the dogs are usually allowed to fast for twenty-four hours before the test.

Active extracts when administered to susceptible animals show three typical effects: First, excitability, then a period of incoördination, followed finally by drowsiness. The second stage of incoördination usually follows in one to two hours; the animal loses control of its legs and of the muscles supporting its head. If nothing occurs to attract the dog's attention, which is absolutely essential, the head will drop, the body sway, and when severely affected, the animal will stagger and fall, the intoxication being peculiarly suggestive and striking. (See Fig. 4.)

The influence of the test dose of the unknown drug is carefully compared with the same dose of the standard administered to another test dog at the same time and 
under the same conditions. When the animals become drowsy, the observations are recorded and the second day following, the two dogs are reversed. The animal previously receiving the test dose of the unknown now receives the test dose of the known, and vice versa, a second observation being made. From the dose of the unknown, selected as produeing the same action as the test dose of the standard, the amount of dilution or concentration that is necessary is determined.

It would hardly occur to the average reader that aside from its epicurean possibilities the frog forms one of the most delicate testing instruments at the disposal of the scientist. Yet it is an actuality that only by careful tests on frogs is it possible to assay the strength of those drugs known as the "heart tonies of the Digitalis Series." These drugs, of which the lily of the valley or convallaria, the fox-glove or digitalis, squill and strophanthus are the most important members, have a special affinity for the heart muscle tissues and are very active poisons. The active principles of all of the drugs are so very much alike that they may be considered as one group and may be physiologically assayed by the same method. They differ greatly, however, in the amount of their activity and in their poisoning powers. It is precisely this poisoning power, particularly with cold-blooded animals, whic is made use of in determining the strength of these drugs. Briefly, the method of administering these poisons and of observing results is as follows: (Fig. 7.)

The drug, or its tincture, is dissolved in salt solution f the same strength as that found in the body fluids. By "pipu " long, slender, glass measuring tubes or "pipettes," having a hypodermio noedle attached, accurately measured amounts are now injected directly through the frog's mouth into the abdominal lymph sac. As a rule, it is necessary to inject several series of about five frogs each for each sample of the drug to be tested. The first series is injected with a standard tincture of strophanthus made up according to the U. S. Pharmacopceia. Usually 0.00015 cubic centimeter per gramme body weight of frog is found to be the smallest amount of this drug who bills the fro be the st is anount "minimum fatal dose" (M. F. D.). The second series is now injected with doses of the unknown varying considerably in size.

After the approximate dose of the poison has been found from the second series, a third series is injected to fix the minimum fatal dose. Finally, a fourth series is injected which fixes the limits of strength very elosely. In a good test, the minimum fatal dose in the fourth series should kill at least three frogs out of five. Now, by comparing the minimum fatel dose of the standard with that of the unto $13 / 4,2 / 3$, etc.

It is usual to express the strength of this series of It is usual to express the strength of this series of drugs in terms of "heart tonic units" (H. T. U.), the
heart tonic unit being ten times the minimum fatal dose per gramme body weight of the frog. The standard tincture of strophanthus has been found to contain 650 heart tonic units. To give the strength of the unknown drug, all that is necessary then is to multiply the above ratio of the minimum fatal doses by 650 to get the number of of H. T. U.'S (heart to

\section{Heat; A Lecture for Beginners}

\section{An Explanation of Terms and Principles Necessary to Understand Engineering Problems}

\section{By Charles H. Bromley}

As THE boiler is a combined heat generator and heat absorber, an understanding of what the unit of heat is, is essential to a thorough understanding of boiler operaion. here are numerous explanations, but they are of little practical value to the power-plant man. One of the most essential things for him to know is that the standard quantity or unit of heat in general use in this country is the British thermal unit (abbreviated B.t.u.). In practice, this quantity is usually considered as that amount of heat required to raise temperature of water $1 \mathrm{deg}$. Fahr. TEMPERATURE.

While the B.t.u. is the quantity of heat, the temperature indicates the quality of heat. One hundred pounds of water at 100 deg. Fahr. contains more heat than one pound of water at 200 degrees. One body of water is twice as hot as the other, but it did not take as much heat to make it that hot as it did to make the larger body one half as hot; see Fig. 1. Remember that temperature indicates only how hot a body is; it does not tell directly how much heat there is in it. which water freezes at 32 degrees and boils at 212 ; the Centigrade, used universally, on which the freezin point is 0 degree and the boiling point 100, and the Reaumur, where the freezing point is 0 degree and the boiling point 80 degrees. We see then that 1 deg. Fahr. $=\frac{5}{9}$ deg. Cent 1 deg. Fahr. = $=4$ deg. Reaumur

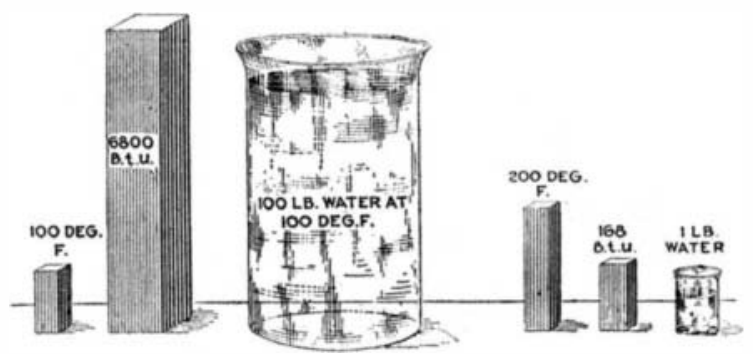

Fig. 1.-Showing that temperature is not alone direct indication of the amount of heat in a body.

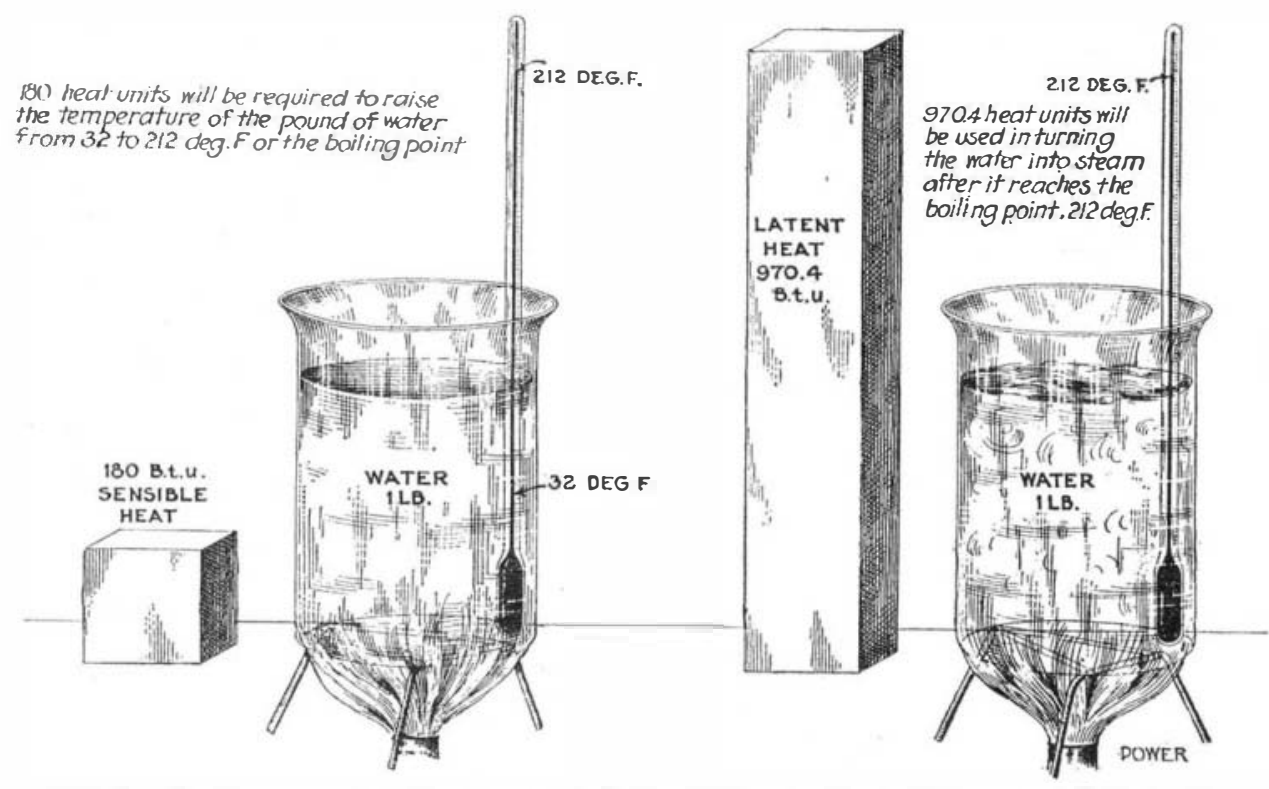

Fig. 2.-Showing the relative amounts of sensible and latent heat needed to boil a pound of water into steam at atmospheric pressure. so that to express the different scales in terms of one another we have:

Fahrenheit degrees $-32 \times 5=$ Centigrade degrees. Fahrenheit degrees $-32 \times \frac{4}{8}=$ Reaumur degrees. Centigrade degrees $\times \frac{9}{5}+32=$ Fahrenheit degrees. Reaumur degrees $\times \frac{9}{4}+32=$ Fahrenheit degrees. ABSOLUTE TEMPERATURE.

Put a cake of ice in a room where the temperature is 20 degrees below zero and the ice will eventually coo off until its temperature is the same as that of the room; the ice will give up heat to the air in the room. We see then that, although ice forms at zero degrees on the Centigrade scale, this is not the lowest temperature that a body may attain; it is not the temperature at which there is a total absence of heat, not absolute zero beyond which it is impossible to cool a body. Neither are the zero points on other thermometers; man puts them where it is convenient, but the absolute zero of temperature is not fixed by man, Nature having established it for man to discover.

Experiments on the expansion of gases kept at a constant pressure show that the volumes and temperatures increase and decrease proportionately; the volume in creasing $\frac{1}{1}$ for each degree Centigrade increase in temperature and decreasing $\frac{1}{5}$ for each degree Centigrade decrease in temperature.

This being so, we see that, mathematically, at a temperature of -273 deg. Cent or - 459.4 deg. Fahr. (the perature of 273 deg. Cont or minus of law, called law, called the Law of Charles, holds true within the limits which we are able to measure. In making calculations in thermodynamics it is sometimes desired to use the absolute zero.

SENSIBLE AND LATENT HEAT.

It is important in boiler practice to know how water is affected by heat. At the temperature of $32 \mathrm{deg}$. Fahr., water changes from a solid to a liquid or from a liquid to a solid; ice forms or melts; it is a critical temperature. To melt one pound of ice at 32 degrees into water at 32 degrees, 144 heat units or B.t.u. must be applied to the ice; and, to freeze one pound at 32 degrees into ice at 32 degrees, 144 B.t.u. must be taken out of the water. To raise the temperature of one pound of water from 32 to 212 degrees, which is the boiling point at atmospheric pressure, 180 B.t.u. must be absorbed, and to cool it from 212 to 32 degrees, 180 B.t.u. must be

Pressure, of course, has a good deal to do with heat and temperature as related to water. For instance, as seen from Fig. 1, there is much more heat in the 100 pounds of water at $100 \mathrm{deg} \mathrm{Fahr}$. than there is in the one pound of water which is at $200 \mathrm{deg}$. Fahr. Suppose we had the one pound of water under 400 pounds absolute pressure, that is, the pressure as indicated by the gage plus the pressure of the atmosphere or 15 pounds; gage plus the pressure of the atmosphere or 15 pounds; pound pound of water would contain 1,208 B.t.u. at this temperature-over seven times as much as when heated to 200 deg. Fahr.

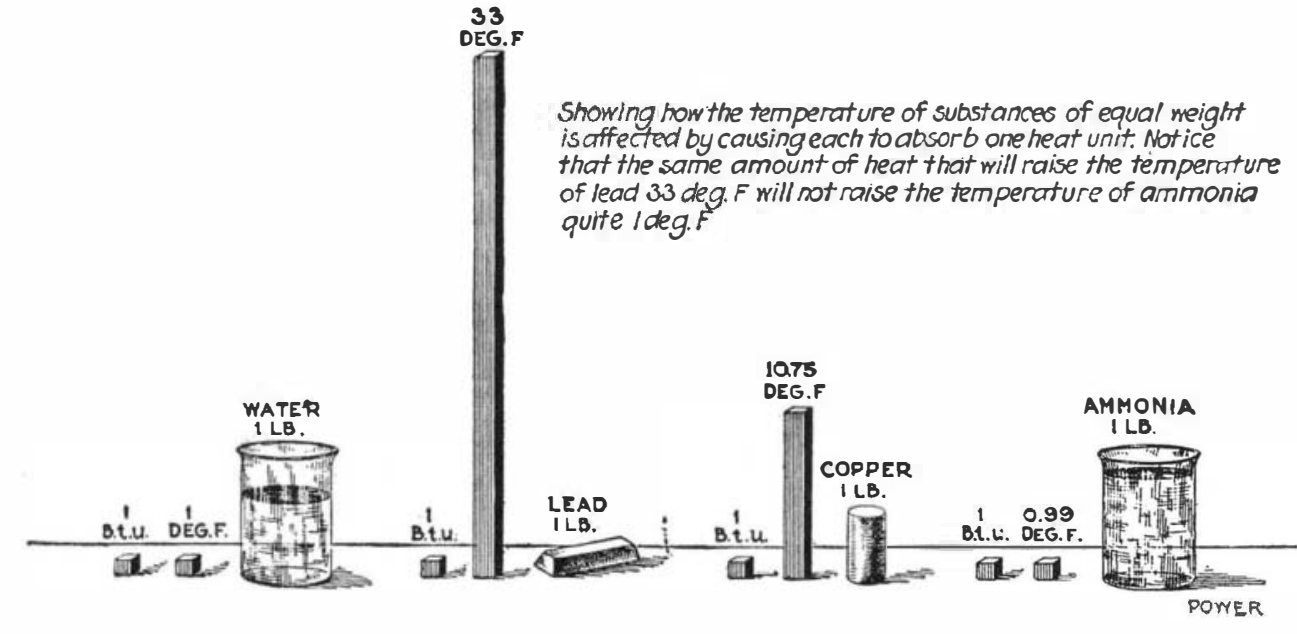

